\title{
Devemos usar beta-bloqueadores para diminuir a progressão dos gliomas como em outras neoplasias (e.g., de mama, ovário e próstata)?
}

Should we use beta-blockers to slow the progression of gliomas as in other neoplasms (e.g., breast, ovary, and prostate)?

\section{Luana Lira de Carvalho Plauto (iD) Rafaela Magalhães de Resende}

Faculdade Pernambucana de Saúde, Recife, PE, Brasil

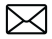

Luana Plauto

luanaplauto@hotmail.com

Editado por:

Juliana Ramos de Andrade

Palavras-chave:

Beta-Antagonistas Adrenérgicos

Glioma

Progressão da Doença

Neoplasias da Mama

Ovário

Próstata
Possuindo uma taxa de sobrevivência de 15 meses após o diagnóstico ${ }^{1}$ e consistindo em $50 \%$ de todos os tumores cerebrais primários ${ }^{2}$, o glioma é um tumor cerebral intra-axial ${ }^{3}$ que possui uma abordagem que vai desde tratamentos conservadores até procedimentos cirúrgicos. As possibilidades de tratamento utilizam de duas vias de ação, o ataque às células cancerígenas propriamente ditas e o combate ao desenvolvimento tumoral através da inibição da angiogênese. ${ }^{4}$ A localização do glioma em meio ao tecido cerebral saudável determina um mau prognóstico, pois, apesar das diversas possibilidades terapêuticas, esse fator dificulta o seu acesso cirúrgico ${ }^{5}$ e favorece um diagnóstico tardio e em estágio avançado. ${ }^{6,7}$ Somado a essa questão, a ação dos fármacos é limitada nesses tumores devido a permeabilidade seletiva da barreira hematoencefálica $(\mathrm{BHE}) .^{8}$

Diante de estudos pré-clínicos que apontam a possível eficácia dos beta-bloqueadores na disseminação de tumores, tais como câncer de mama, ovário e próstata9-11, foi colocada em questão a eficácia desses fármacos no tratamento de glioma. $\bigcirc$ grande número de pessoas afetadas pela doença e a possibilidade de eficácia da droga despertaram o interesse em evidenciar a aplicabilidade da terapia medicamentosa dos beta-bloqueadores em pacientes com glioma. Com esse objetivo, o artigo "Beta-blockers and glioma: a systematic review of preclinical studies and clinical results"12 foi desenvolvido por meio de uma revisão sistemática utilizando de dados extraídos de 11 estudos, sendo 10 deles pré-clínicos e um clínico. Ishaan Ashwini Tewarie e os coautores ${ }^{12}$ identificaram nos estudos pré-clínicos quatro mecanismos que explicam a ação em potencial dos beta-bloqueadores no crescimento desses tumores.

O primeiro mecanismo consiste na redução da proliferação através da inibição por via do $\mathrm{AMPc}$, tanto pelo bloqueio da atividade ligada ao substrato como por sua ação de agonista inverso, além da redução dose-dependente do adenilato ciclase. ${ }^{12}$ 
A segunda questão identificada é o decréscimo da migração celular, sendo evidenciado que a administração de beta-agonistas é capaz de aumentar a propensão à metástases, enquanto o uso de beta-bloqueadores reduz a migração celular, bem como a ruptura da barreira hemato-encefálica (BHE). ${ }^{12}$

A associação dos beta-bloqueadores com outras drogas, mediante o aumento da sensibilidade aos fármacos, é o terceiro mecanismo. Esse acréscimo ocorre devido à inibição de substâncias que atuam no efluxo de drogas através da barreira hematoencefálica, conhecidas como p-glicoproteínas, capazes de reduzir a ação dessas no sistema nervoso central. Outros estudos evidenciam essa ação apontando o carvedilol como o fármaco mais potente entre esses fármacos. ${ }^{12}$

O último mecanismo apontado é a indução da morte celular, embora os meios pelos quais essa apoptose ocorra permaneçam sem elucidação. ${ }^{12}$

Apesar de evidenciados como capazes de atuar efetivamente no tratamento dos gliomas pelos estudos pré-clínicos, os beta-bloqueadores não apresentaram efeito relacionado ao aumento de sobrevida no estudo observacional. Tal diferença entre o cenário teórico e prático ressalta a necessidade de aprofundamento a respeito dos mecanismos de ação dessas drogas, principalmente sobre os possíveis desfechos do seu uso na prática clínica do tratamento dos gliomas.

Diante desse cenário, permanece a busca por alternativas que permitam a superação dos atuais entraves no tratamento dessa neoplasia. Métodos como a ruptura por ultrassom pulsado e o tratamento por nanopartículas surgem como possibilidades diante das limitações à ação medicamentosa devido a seletividade da BHE. O uso de ultrassom pulsado obteve resultados satisfatórios em estudos pré-clínicos, demonstrando eficácia no aumento da concentração das drogas e também retardo do crescimento tumoral. ${ }^{13}$ Já o potencial do tratamento por nanopartículas consiste nas variações de dimensões das biomoléculas e de material de revestimento, capazes de permitir uma interação com células humanas para ultrapassar a barreira e, consequentemente, atuar como ligandos específicos para células tumorais como as do glioma. ${ }^{14}$ Outra perspectiva de tratamento é encontrada nas terapias moleculares específicas, que objetivam atingir as vias oncogênicas dos gliomas interagindo com seus receptores ou alvos ajusantes. ${ }^{15,16}$

Assim como foi observado no artigo acerca do uso de beta-bloqueadores e em tantos outros presentes na literatura, a baixa evidência clínica limita o uso de terapias alternativas que buscam gerar melhores prognósticos no tratamento de gliomas. Sendo assim, o aprofundamento de estudos, como o abordado no presente comentário, é fundamental para proporcionar o aumento da sobrevida dos pacientes afetados pelo glioma.
Contribuição dos autores: As autoras contribuiram de forma igual.

Luana Lira de Carvalho Plauto

https://orcid.org/0000-0003-2285-0156

Rafaela Magalhães de Resende

https://orcid.org/0000-0003-0147-2387

\section{Referências}

1. Joaquim A, Almeida JPC, Ghizoni E, Valadares MC and Tedeschi H. Manejo dos Pacientes com Gliomas de Alto Grau. J Bras Neurocirurg 2014;25(1):61-73 Doi: $10.3747 /$ co. 23.3082

2. Santos AL. Gliomas, tumores malignos que surgem no sistema nervoso. Ciências e Educação 2021;7(2): 12-12 Doi: 10.51891/rease.v7i2.583

3. Rapalino O, Batchelor T and González RG. Intra-axial brain tumors. Handb Clin Neurol 2016;135:253-274 Doi:10.1016/b978-0-444-53485-9.00014-3

4. Alves PMG. Abordagem Terapêutica de Gliomas Cerebrais de Alto Grau. Covilhã: Universidade da Beira Interior; 2014.Disponível em: https://ubibliorum.ubi.pt/ bitstream/10400.6/4864/1/3359_6744.pdf

5. Kirby AJ and Finnerty GT. New strategies for managing adult gliomas. J Neurol 2021;268(10):3666-3674 Doi: 10.1007/s00415-020-09884-3

6. Arnautovic A, Billups C, Broniscer A, Gajiar A, Boop $F$ and Qaddoumi I. Delayed diagnosis of childhood low-grade glioma: causes, consequences, and potential solutions. Childs Nerv Syst 2015;31(7):1067-1077 Doi:10.1007/s00381-015-2670-1

7. Aggarwal A, Herz N, Campbell P, Arkush L, Short S and Rees J. Diagnostic delay and survival in high-grade gliomas - evidence of the 'waiting time paradox'? $\mathrm{Br} J$ Neurosurg 2015;29(4):520-523 Doi:10.3109/02688 697.2015 .1012050

8. Dai H, Marbach $P$, Lemaire $M$, Hayes $M$ and Elmquist WF. Distribution of STI-571 to the brain is limited by P-glycoprotein-mediated efflux. J Pharmacol Exp Ther 2003;304(3):1085-1092 Doi:10.1124/ ipet. 102.045260

9. Barron TI, Sharp L and Visvanathan K. Beta-adrenergic blocking drugs in breast cancer: a perspective review. Ther Adv Med Oncol 2012;4(3):113-125 Doi: $10.1177 / 1758834012439738$

10. Baek MH, Kim DY, Kim SO, Kim YJ and Park YH. Impact of beta blockers on survival outcomes in ovarian cancer: a nationwide population-based cohort study. J Gynecol Oncol 2018;29(6):e82 Doi:10.3802/igo.2018.29.e82

11. Zahalka AH, Fram E, Lin W, Mohn L, Frenette PS, Agalliu I and Watts KL. Use of beta-blocker types and risk of incident prostate cancer in a multiethnic population. Urol On 
col 2020;38(10):794.e711-794.e716 Doi:10.1016/i. urolonc. 2020.03 .024

12. Tewarie IA, Senders JT, Hulsbergen AFC, Kremer S and Broekman MLD. Beta-blockers and glioma: a systematic review of preclinical studies and clinical results. Neurosurg Rev 2021;44(2):669-677 Doi:10.1007/s 10143 020-01277-4

13. Bachmakov I, Werner U, Endress B, Auge D and Fromm MF. Characterization of beta-adrenoceptor antagonists as substrates and inhibitors of the drug transporter P-glycoprotein. Fundam Clin Pharmacol 2006;20(3):273-282 Doi:10.1111/j.1472-8206.2006.00408.x

14. Carpentier A, Canney M, Vignot A, Reina V, Beccaria K,
Horodyckid C, ... Idbaih A. Clinical trial of blood-brain barrier disruption by pulsed ultrasound. Sci Transl Med 2016;8(343):343re342 Doi:10.1 126/scitrans/med.aaf6086

15. Lopes JC and Torres MLP. Utilização de nanopartículas no tratamento do câncer: aspectos gerais, mecanismos de ação antineoplásicos e aplicabilidades tumorais. Rev Bras Cancerol 2019;65(4):e-13400 Doi:0.32635/2176. 9745.RBC.2019v65n4.400

16. Kipper F. Novas abordagens terapêuticas para glioblastoma baseadas no ensaio de resposta a terapias em culturas derivadas de pacientes. Porto Alegre: UFRGS; 2017.Disponível em: https://www.lume.ufrgs.br/handle/10183/170182 